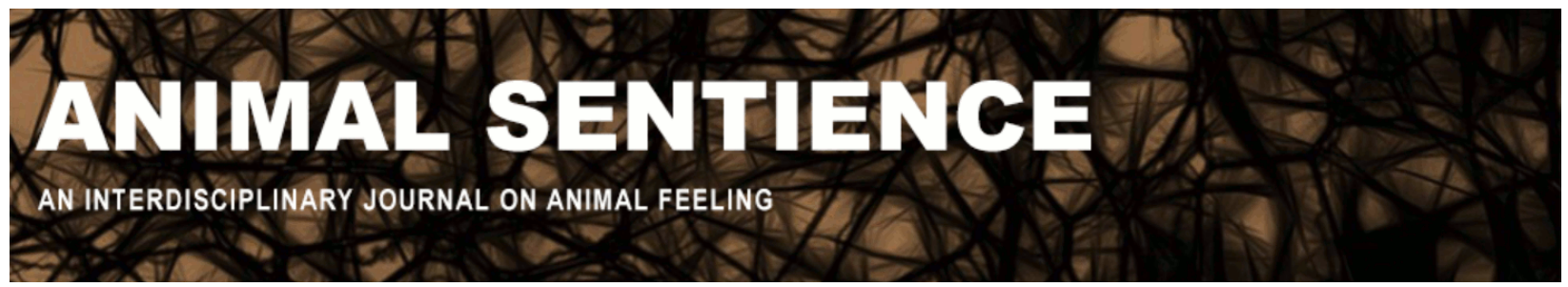

Denson, Thomas F. (2018) Inferring emotion from amygdala activation alone is problematic. Animal Sentience 22(9)

DOI: $10.51291 / 2377-7478.1337$

Date of submission: 2018-05-27

Date of acceptance: 2018-06-01

(c) (†)

This article has appeared in the journal Animal

Sentience, a peer-reviewed journal on animal

cognition and feeling. It has been made open access,

free for all, by WellBeing International and deposited

in the WBI Studies Repository. For more information,

please contact

wbisr-info@wellbeingintl.org.

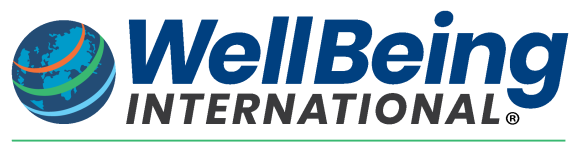

SOLUTIONS FOR PEOPLE, ANIMALS AND ENVIRONMENT 


\title{
Inferring emotion from amygdala activation alone is problematic
}

\author{
Commentary on Cook et al. on Dog Jealousy
}

\author{
Thomas F. Denson \\ School of Psychology, University of New South Wales
}

\begin{abstract}
Cook et al. investigated neural responses in domestic dogs in an experiment designed to elicit jealousy. Relative to a control condition, watching the dogs' caregivers feed a fake dog activated the amygdala bilaterally. Dogs rated higher in dog-directed aggressiveness showed larger initial amygdala activation. Amygdala activity in this context is insufficient evidence to infer that the dogs experienced jealousy or even negative affect. The experimental design does not provide an adequate level of control to infer the presence of jealousy.
\end{abstract}

Tom Denson is associate professor of psychology at the University of New South Wales in Sydney, Australia. He is an experimental social-personality psychologist. His primary interests are anger and aggression, social neuroscience, and social neuroendocrinology. He has conducted several functional magnetic resonance imaging studies of anger and aggression in humans. Website

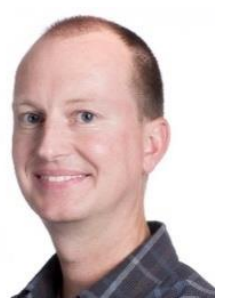

Jealousy, anger, and aggressive behavior may strain human-canine relations. Thus, research into understanding the emotional lives of domestic dogs will likely improve human-canine interactions and is therefore an important endeavor. In a very clever experiment, Cook et al. (2018) have conducted an investigation into the neural correlates of jealousy in dogs. Using functional magnetic resonance imaging ( $\mathrm{FMRI}$ ), 13 domestic dogs were trained for the MRI environment and completed three runs of three trial types: dog receives food, dog sees fake dog get food, and dog sees food deposited in a bucket (i.e., no one gets food). Analyses focused on the dog-directed aggression scale of the Canine Behavioral Assessment and Research Questionnaire (C-BARQ) as an additional predictor of BOLD responses in the bilateral amygdala.

Despite my enthusiasm for the topic and the general aims of this research, the claims are not justified by theory or the data. On a theoretical level, it seems unlikely that dogs experience jealousy in the same way humans do, if at all. Dogs certainly experience happiness, fear, anger, disgust, surprise, and sadness, which belong to a class of emotions known as basic emotions (Ekman, 1992). These six emotions are shared with many mammalian species. However, jealousy is part of a class of emotions known as self-conscious emotions or social emotions (Tangney \& Salovey, 1999). These emotions are generally thought to be restricted to the human experience because they require higher-order abstract cognition such as theory of mind, complex cognitive appraisals, and attributions. Similarly, in human laboratory experiments, jealousy is invoked by threatened self-esteem (DeSteno, Valdesolo, \& Bartlett, 2006). Dogs would require self-consciousness to experience a similar pathway to jealousy.

Examining just one brain structure, the amygdala, is highly unlikely to provide evidence for any specific emotional experience. The authors are right to qualify their findings by noting that amygdala responsivity is not isomorphic with any specific emotional experience. A meta- 
analysis of human neuroimaging studies showed that all emotional stimuli increased amygdala activation, including positive emotions (Costafreda, Brammer, \& David, \& Fu, 2008). Thus, the amygdala can be viewed more as a brain structure that responds to stimuli with emotional relevance regardless of emotional valence. With that in mind, examining only the amygdala is insufficient to make claims about jealously. Based on amygdala activity alone, it is equally possible to claim that the dogs were happy or sad to see the fake dog get a treat, or that they simply found that condition more emotionally relevant than the others without experiencing any emotion at all.

Neuroimaging in humans has become increasingly detailed in its description of brain networks involved in different emotions. During these early days of research into emotions and the brain in dogs, an exploratory whole-brain analysis could be helpful in guiding future research and so could functional connectivity analyses. Future work could examine more widespread brain networks activated during emotions of interest to make stronger claims about the emotional experiences of dogs.

My assessment of this research is aligned with that of Vonk (2018), who suggested that more evidence with additional control conditions is needed before one can ascribe jealousy to dogs. The authors did not provide any convincing data on whether the subjects actually perceived the fake dog to be real. An unmoving statue that smells like another dog could be perceived as strange and hence threatening in its own right initially. In fact, the dogs who were rated highest on the C-BARQ aggression measure (i.e., those dogs highest in threat sensitivity) showed the largest amygdala habituation response. This finding suggests that they may have quickly figured out the charade. The finding that no dogs showed any aggression toward the fake dog is also consistent with this possibility. Jealousy is a robust elicitor of aggression in humans.

Assuming that the subjects did indeed perceive the fake dog as real, the experiment requires a control condition in which the fake dog is fed by someone other than the caregiver. Such a condition would reflect the emotion of envy in humans (e.g., coveting what someone else has). If something akin to a jealousy response is elicited, one should only see relevant BOLD responses in the caregiver condition. If relevant neural responses are observed in both conditions, the result is probably more indicative of intraspecific resource competition (e.g., something more akin to envy). The current design does not distinguish between these two possibilities.

One is left with the difficult problem of how to detect jealousy in dogs, if it exists. Detecting jealousy nonverbally is not easy in humans. Unlike basic emotions, jealousy does not have a universal facial expression. The only reliable method with people is to ask - and even then, humans must be willing and able to report their emotional experience. To date, there is no strong evidence for a unique pattern of brain activity during experienced jealousy, suggesting that finding the neural correlates of jealousy in dogs may take some time.

A final issue is that five of the 13 dogs had the lowest aggression score possible. Thus, the results rest on just 8 dogs. Replication and extension with a larger sample is warranted.

In sum, this experiment is part of growing methodological advances taking place in dog imaging, and I commend the authors for the effort. Learning as much as we can about the emotional lives of domestic dogs will no doubt improve human-canine relations and canine welfare. 


\section{References}

Cook, P., Prichard, A., Spivak, M., \& Berns, G. S. (2018). Jealousy in dogs? Evidence from brain imaging. Animal Sentience 22(1).

Costafreda, S. G., Brammer, M. J., David, A. S., \& Fu, C. H. (2008). Predictors of amygdala activation during the processing of emotional stimuli: A meta-analysis of 385 PET and fMRI studies. Brain Research Reviews, 58(1), 57-70.

DeSteno, D., Valdesolo, P., \& Bartlett, M. Y. (2006). Jealousy and the threatened self: Getting to the heart of the green-eyed monster. Journal of Personality and Social Psychology, 91(4), 626-641.

Ekman, P. (1992). An argument for basic emotions. Cognition and Emotion, 6, 169-200.

Tangney, J. P., \& Salovey, P. (1999). Problematic social emotions: Shame, guilt, jealousy, and envy. Washington, DC: American Psychological Association.

Vonk, J. (2018). Researchers, not dogs, lack control in an experiment on jealousy. Animal Sentience 22(2). 


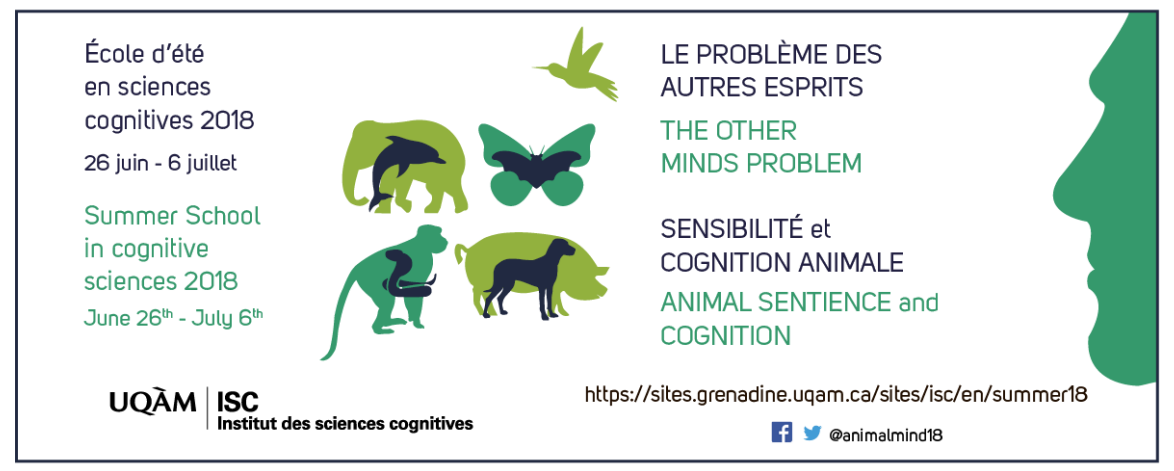

\section{ISC 2018 Summer School in Cognitive Sciences}

June 26 - July 6, 2018

Montreal (Canada)

The Other Minds Problem:

Animal Sentience and Cognition

Overview. Since Descartes, philosophers know there is no way to know for sure what - or whether - others feel (not even if they tell you). Science, however, is not about certainty but about probability and evidence. The 7.5 billion individual members of the human species can tell us what they are feeling. But there are 9 million other species on the planet (20 quintillion individuals), from elephants to jellyfish, with which humans share biological and cognitive ancestry, but not one other species can speak: Which of them can feel - and what do they feel? Their human spokespersons - the comparative psychologists, ethologists, evolutionists, and cognitive neurobiologists who are the world's leading experts in "mind-reading" other species - will provide a sweeping panorama of what it feels like to be an elephant, ape, whale, cow, pig, dog, chicken, bat, fish, lizard, lobster, snail: This growing body of facts about nonhuman sentience has profound implications not only for our understanding of human cognition, but for our treatment of other sentient species.

Gregory Berns: Decoding the Dog's Mind with Awake Neuroimaging Gordon Burghardt: Probing the Umwelt of Reptiles

Jon Sakata: Audience Effects on Communication Signals

PANEL 1: Reptiles, Birds and Mammals

WORKSHOP 1: Kristin Andrews: The "Other" Problems: Mind,

Behavior, and Agency

Sarah Brosnan: How Do Primates Feel About Their Social Partners?

Alexander Ophir: The Cognitive Ecology of Monogamy

Michael Hendricks: Integrating Action and Perception in a Small

Nervous System

PANEL 2: Primates, Voles and Worms

WORKSHOP 2: Jonathan Birch: Animal Sentience and the

Precautionary Principle

Malcolm Maclver: How Sentience Changed After Fish Invaded Land 385 Million Years Ago

Sarah Woolley: Neural Mechanisms of Preference in Female Songbird

Simon Reader: Animal Social Learning: Implications for Understanding Others

PANEL 3: Sea to Land to Air

WORKSHOP 3: Steven M. Wise: Nonhuman Personhood

Tomoko Ohyama: Action Selection in a Small Brain (Drosophila

Maggot)

Mike Ryan: "Crazy Love": Nonlinearity and Irrationality in Mate Choice

Louis Lefebvre: Animal Innovation: From Ecology to Neurotransmitters

PANEL 4: Maggots, Frogs and Birds: Flexibility Evolving

SPECIAL EVENT: Mario Cyr: Polar Bears

Colin Chapman: Why Do We Want to Think People Are Different?

Vladimir Pradosudov: Chickadee Spatial Cognition

Jonathan Balcombe: The Sentient World of Fishes

PANEL 5: Similarities and Differences

WORKSHOP 5 (part 1): Gary Comstock: A Cow's Concept of Her Future WORKSHOP 5 (part 2): Jean-Jacques Kona-Boun: Physical and Mental Risks to Cattle and Horses in Rodeos

Joshua Plotnik: Thoughtful Trunks: Application of Elephant Cognition

for Elephant Conservation
Lori Marino: Who Are Dolphins?

Larry Young: The Neurobiology of Social Bonding, Empathy and Social Loss in Monogamous Voles

Panel 6: Mammals All, Great and Small

WORKSHOP 6: Lori Marino: The Inconvenient Truth About Thinking Chickens

Andrew Adamatzky: Slime Mould: Cognition Through Computation Frantisek Baluska \& Stefano Mancuso: What a Plant Knows and Perceives

Arthur Reber: A Novel Theory of the Origin of Mind: Conversations With a Caterpillar and a Bacterium

PANEL 7: Microbes, Molds and Plants

WORKSHOP 7: Suzanne Held \& Michael Mendl: Pig Cognition and Why It Matters

James Simmons: What Is It Like To Be A Bat?

Debbie Kelly: Spatial Cognition in Food-Storing

Steve Phelps: Social Cognition Across Species

PANEL 8: Social Space

WORKSHOP 8: To be announced

Lars Chittka: The Mind of the Bee

Reuven Dukas: Insect Emotions: Mechanisms and Evolutionary Biology Adam Shriver: Do Human Lesion Studies Tell Us the Cortex is Required for Pain Experiences?

PANEL 9: The Invertebrate Mind

WORKSHOP 9: Delcianna Winders: Nonhuman Animals in Sport and Entertainment

Carel ten Cate: Avian Capacity for Categorization and Abstraction

Jennifer Mather: Do Squid Have a Sense of Self?

Steve Chang: Neurobiology of Monkeys Thinking About Other

Monkeys

PANEL 10: Others in Mind

WORKSHOP 10: The Legal Status of Sentient Nonhuman Species 